\title{
Regression of plaque burden after primary percutaneous coronary intervention (PCI) in a patient with TVD: 4 years follow-up
}

\author{
AHM W Islam ${ }^{1}$, S Munwar ${ }^{2}$, S Talukder ${ }^{3}$, A Q M Reza ${ }^{4}$, T Ahmed $^{5}$
}

\begin{abstract}
Coronary Artery disease (CAD) is an important cause of mortality and morbidity in the developed world as well as in Bangladesh. Treatment of Acute Myocardial Infarction (AMI) patient either by Streptokinase (STK) or Primary Percutaneous Coronary Intervention ( $\mathrm{pPCI}$ ) has increased the survival outcome and reduced the mortality. Several studies have documented the significant beneficial role pPCI in terms of in-hospital survival outcome over thrombolysis. Our patient, who had Anterior MI in 2004 and his CAG revealed TVD. pPCI of the culprit mid LAD lesion with Bare Metal Stent (BMS) was done immediately after hospitalization. He was later referred for $\mathrm{CABG}$, but decision was postponed because of asymptomatic status. His re-look CAG on 20-02-2008 (i.e., 4 yrs after the original procedure), revealed patent LAD stent with the regression of atherosclerotic plaque in Ostio-Proximal LAD and proximal LCX. Our findings indicated that pPCI with rigid control of $\mathrm{CAD}$ risk factors and modification of lifestyle plays a key role in the regression of atherosclerotic plaque and maintenance of stent patency.
\end{abstract}

Correspondence and Reprint request: Dr. A H M Waliul Islam, Dept of Interventional and Invasive cardiology, Apollo Hospitals Dhaka, Bangladesh.Ahmwislam@apollodhaka.com

Introduction: Acute coronary syndrome (ACS) is one of the important causes of death in the developing as well as developed world. Managing the patient either by STK or pPCI can reduce the mortality and improve 30 days inhospital survival out come. ${ }^{1}$ Primary angioplasty is the preferred treatment modality for AMI in centers with available cath lab facilities and well-trained personnel. ${ }^{2}$ The main advantage of pPCI is the achievement of a higher rate of coronary recanalization with a lower risk of intracranial bleeding. ${ }^{3-5}$ Now a days pPCI has become the choice of revascularization procedure compared to thrombolysis, for establishing TIMI III distal flow. It has already been established that $\mathrm{pPCI}$ is superior to thrombolytic therapy for the treatment of patient with STEMI. Many studies have demonstrated that pPCI within 2 hrs of onset of symptoms has lower mortality and higher rate of complete reperfusion. ${ }^{6}$ Our present case shows the survival outcome in patient with acute Ant MI with TVD who had pPCI in Mid LAD in the year 2004.

Case: 61 yr Bangladeshi gentleman admitted to a local hospital with the onset of sudden chest pain for one and half hrs. His admission ECG revealed acute ST elevation in anterior chest leads (Fig.1).

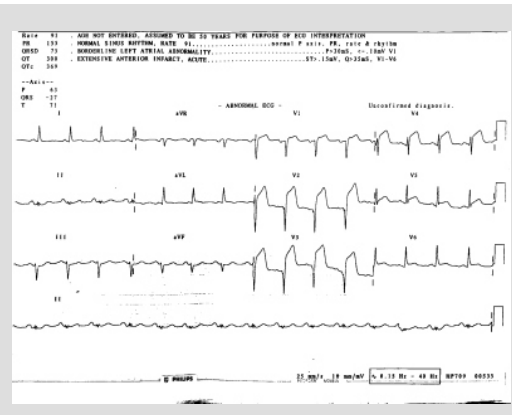

Figure 1: ECG before Primary PCI in 2004 during chest pain showing Acute ST elevation in Anterior Chest leads
He is a known hypertensive on medication and active smoker with no known positive family history for CAD. He was taken to the cardiac cath lab and CAG revealed TVD (Ostial + mid LAD, tight distal LCX and 100\% RCA) with the culprit lesion in the mid LAD (Fig. 2).

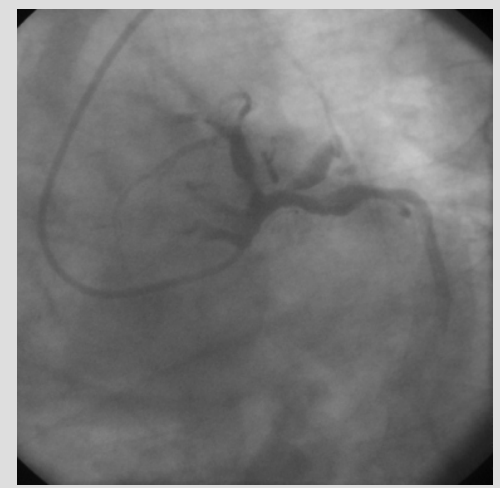

Figure 2a: Culprit lesion in Mid LAD

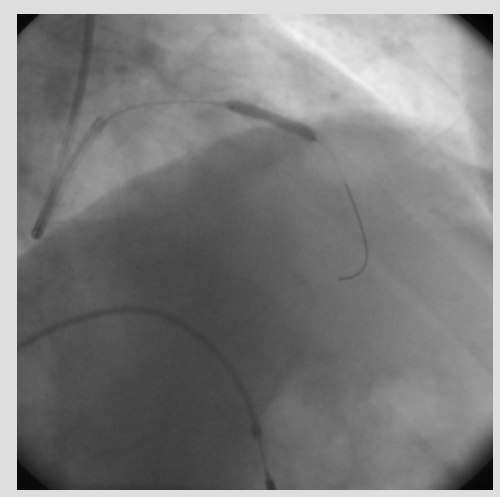

Figure 2b: Stenting of mid LAD lesion 


\section{Regression of plaque}

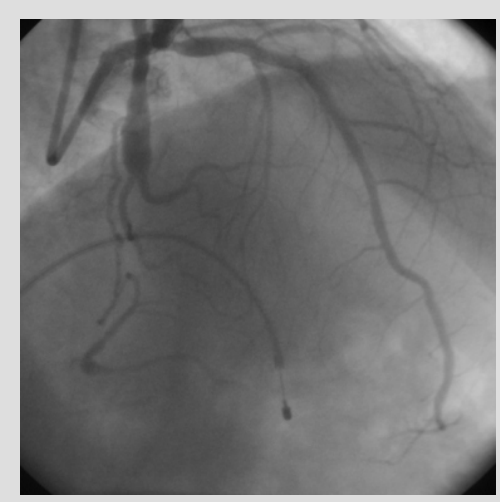

Figure 2c: Post PCI

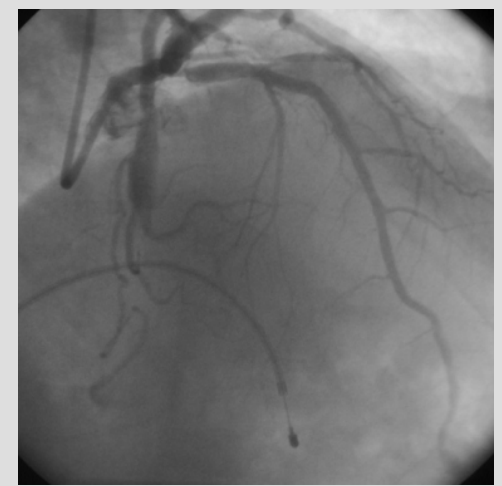

Figure 2d: Final cine showed no residual lesion

Figure 2: Shows culprit lesion in mid LAD and subsequent opening with TIIMI III distal flow by primary PCI in 2004

Emergency pPCI to culprit lesion was done with a BMS of $3 \mathrm{x}$ $19 \mathrm{~mm}$ size deployed at 14ATM and post dilated with $3.5 \times 12$ $\mathrm{mm}$ balloon at 12ATM. Final CAG showed no residual stenosis with achievement of TMI III distal flow. Post procedural ECG showed almost normalization of Elevated ST in the anterior chest leads (Fig.3).

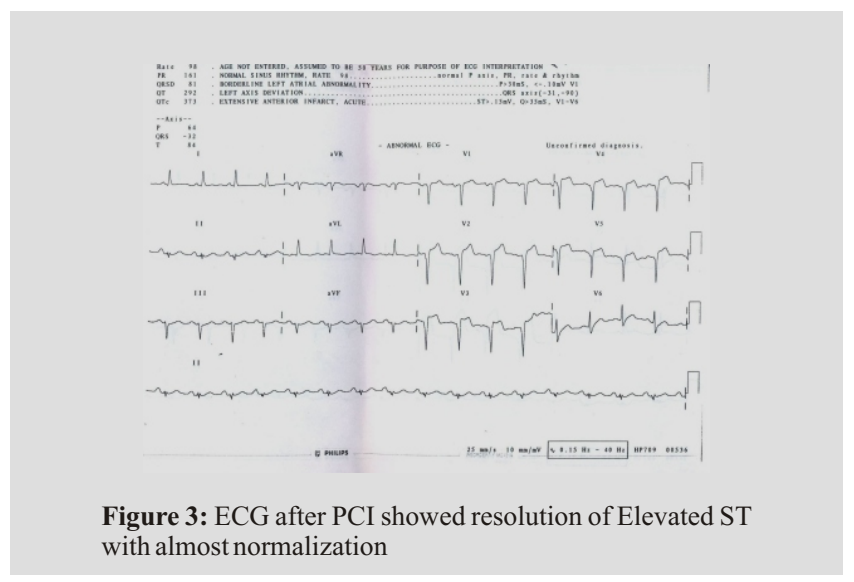

He was discharged in a stable condition and given beta blocker, losartan plus HTZ, atorovastatin with nicotinic acid, Clopidegrol plus aspirin. He was followed-up in the OPD with the continuation of medication, regular exercise, and diet control. He stopped smoking. He remained asymptomatic throughout. He did not attend OPD regularly, but otherwise remained compliant. In 2007, he was advised for SPECT perfusion scan for routine follow-up. SPECT perfusion revealed MI involving apical wall with a strip of per-infarct hibernation and also showed myocardial hibernation in septum (Fig.4.).

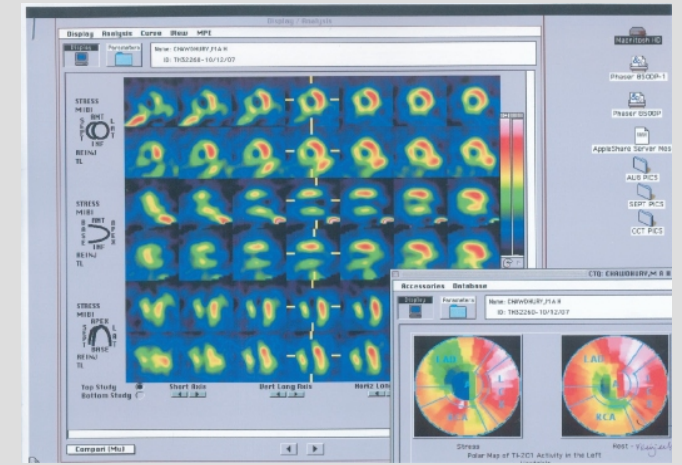

Figure 4: ECG after PCI showed resolution of Elevated ST with almost normalization

He was planned for a re-look CAG to evaluate his coronary status. This revealed, a patent LAD stent and regression of Ostio-proximal LAD plaque (Fig.5) (Fig.6).

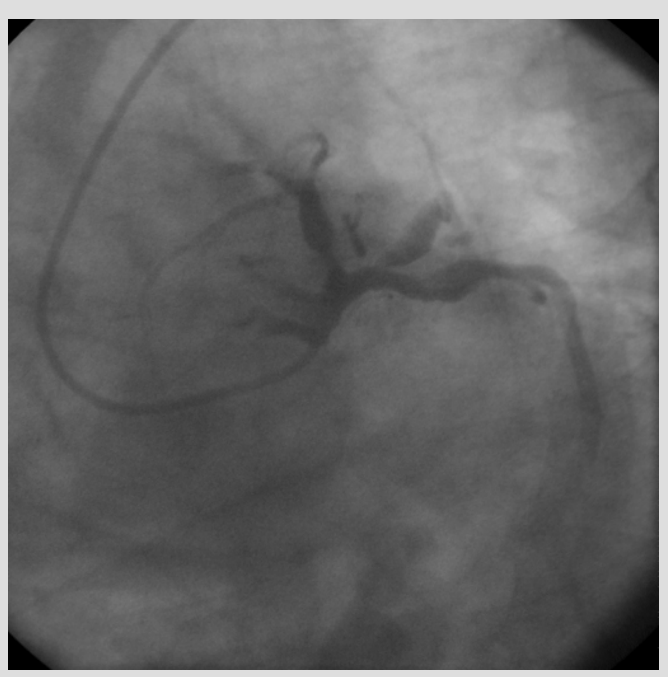

Figure 5a: Ostial LAD and OM1 Plaque in 2004 


\section{Regression of plaque}

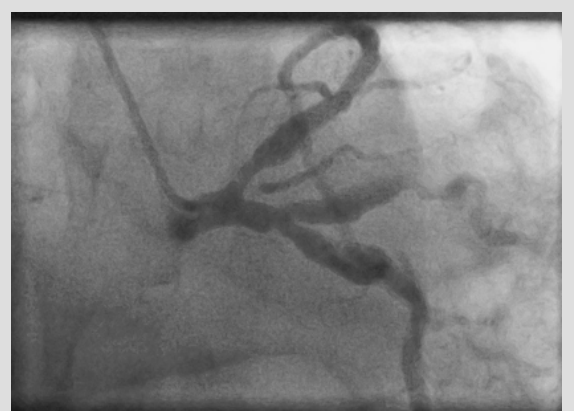

Figure 5b: Regression of same territory in 2008

Figure 5: Arrow indicated the regression of Ostio-Proximal LAD plaque

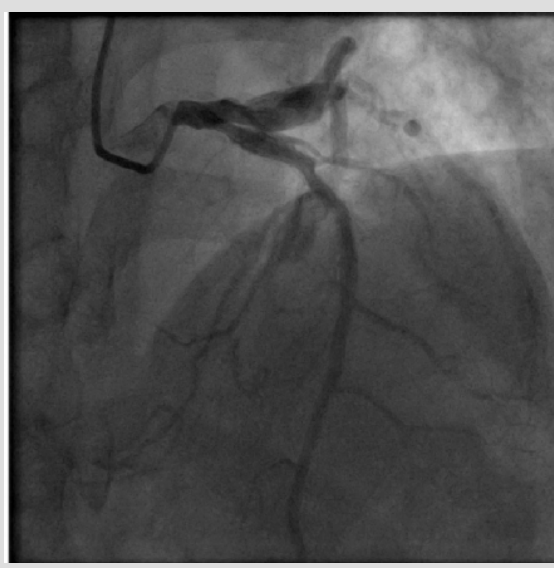

Figure 6a: Re-look CAG 2008

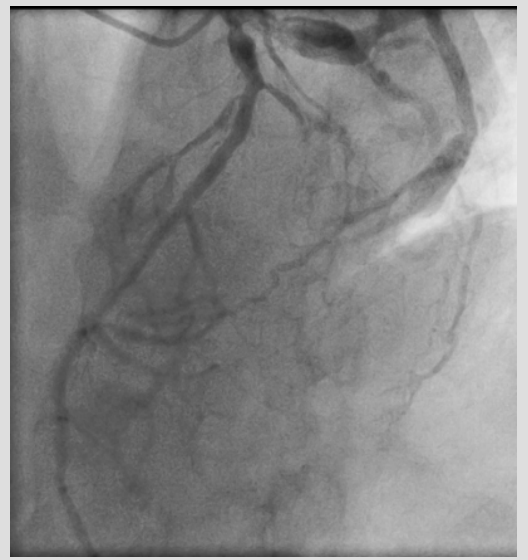

Figure 6b: Re-look CAG 2008

Figure 6: Arrow indicated patent Proximal LAD Stent with good distal runoff
Slow flow in the circ and diffuse small vessel disease was noted, explaining SPECT perfusion findings. He was discharged in a stable condition with the advice of continuing medication, life style modification and regular follow-up.

Discussion: It has been well established that the survival outcome of patients with Acute Myocardial Infarction by primary $\mathrm{PCI}$ is superior to conventional thrombolytic therapy. ${ }^{7}$ Weaver et al., ${ }^{8}$ demonstrated that pPCI results in reduced rates of mortality, reinfarction, and stroke. It has been well documented that PTCA alone in AMI, recurrence of ischaemia or re-infarction occurs in $37-49 \%$ and late infarct related artery re-occlusion in $9-14 \%$. Stone has demonstrated that pPCI (stenting) has low rates of in-hospital death $(0.8 \%)$, re-infarction (1.7\%), recurrent Ischaemia 3.8\% and pre-discharge target vessel revascularization for ischaemia (3.8\%). ${ }^{1}$ In patient undergoing pPCI, procedural success provides significant prognostic values. ${ }^{4,8}$ Patient selection, lesion size, vessel caliber and plaque burden, overall door-to balloon time are the key factors in the procedural success and in-hospital 30 days survival outcome. ${ }^{1}$ Many have demonstrated that pPCI reduces the risk of left ventricular free wall rupture. ${ }^{9-10}$ Many have demonstrated that the better survival outcome among patients treated with pPCI, even when need to be transported to a center with cath lab facilities. ${ }^{6,11-12}$ Considering the better survival outcome and less procedural complications and decreasing morbidity, the UK government is considering to establish a national primary angioplasty service for patients with acute myocardial infarction. ${ }^{13}$ Our present patient demonstrated that the rigid control of CAD risk factors by regular exercise, complete withdrawal of smoking and antiplatelet and lipid lowering agents are not only the key factors in the maintenance of stent patency, but can reduce plaque burden. Nicholls et al. ${ }^{14}$ has described male gender, diabetes, and a history of prior revascularization are strong independent predictors of atherosclerotic burden in coronary disease patients. Birgelen $\mathrm{CV}$ et al. $^{15}$ documented that positive linear relation between LDL cholesterol and annual changes in plaque size, with an LDL value of $75 \mathrm{mg} / \mathrm{dL}$ predicting, on average, no plaque progression. HDL cholesterol shows an inverse relation with annual changes in plaque size. In the landmark COURAGE study of patient with Stable Angina, revealed that PCI in addition to optimal medical therapy reduced the prevalence of angina. ${ }^{16}$ Therefore, our present patient's strong compliance to life style modification and rigid cholesterol control by lipid lowering agent, may have caused regression of plaque burden.

\section{References:}

1. Stone GW, Brodie BR, Griffin JJ. Prospective, multicenter study of the safety and feasibility of primary stenting in acute myocardial infarction:in-hospital and 30 day results of the PAMI pilot trial. J Am Coll Cardiol. 1998;31:23-30.

2. Aros F, Loma-Osorio AA, Aloso JJ. Guias de la sociedad espala de 


\section{Regression of plaque}

cardiologia del manejo del infart de miocardio. Rev Esp Cardiolo. 1999;52:919-56.

3. Grines CL, Browne KF, Marco J. For the primary angioplasty in myocardial infarction study group: a comparison of immediate angioplasty with thrombolytic therapy for acute myocardial infarction. $\mathrm{N}$ Eng J Med. 1993;328:673-9.

4. Zijlstra F, de Boer MJ, Hoorntje JCA. A comparison of immediate coronary angioplasty with intravenous streptokinase in acute myocardial infarction. NEng J Med. 1993;328:680-4.

5. Gracia E, Elizaga J, Perez N. Primary angioplasty versus systemic thrombolysis in anterior myocardial infarction. J Am Coll Cardiol. 1999;33:605-11.

6. Anderson HR, Nielson TT, Rasmussen K. A comparison of coronary angioplasty with fibrinolytic therapy in acute myocardial infarction. $\mathrm{N}$ Eng J Med. 2003;349:733-42.

7. Henriques JPS, Zijlistra F, Hof AWJV. Primary percutaneous coronary intervention versus thrombolytic treatment: long term follow up according to infarct location. Heart. 2006;92:75-79.

8. Weaver WD, Simes RJ, Betriu A. Comparison of primary coronary angioplasty and intravenous thrombolytic therapy for acute myocardial infarction: a qualitative review. JAMA. 1997;278:2093-8.

9. Moreno R, Lopez-Sendon J, Gracia E. Primary angioplasty reduces the risk of left ventricular free wall rupture compared with thrombolysis in patients with acute myocardial infarction. J Am Coll Cardiol. 2002;39:598-603.
10. Reddy SG, Roberts WC. Frequency of rupture of LV free wall or ventricular septum among necropsy case of fatal acute myocardial infarction since introduction of coronary care units. Am. J Cardiol. 1989;63:906-11.

11. Madsen JK, Grande P, Saunamaki K. DNAMI-2 Investigators. A comparison of coronary angioplasty with fibrinolytic therapy in acute myocardial infarction. N Eng J Med. 2003;349:733-42.

12. Widimisky P, Groch L, Zelizko M . Multicenter randomized trial comparing transport to primary PCI versus immediate thrombolysis vs combined strategy for patients with acute myocardial infarction presenting to a community hospital without catheterization laboratory. The PRAGUE study. Eur H J. 2000;21:823-31.

13. Smith D. Primary angioplasty should be the first line treatment for acute myocardial infarction. BMJ. 2004;328:1254-7.

14. Nicholls SJ, Tuzcu EM. Relationship between cardiovascular risk factors and atherosclerotic disease burden measured by intravascular ultrasound. J Am Coll Cardiol. 2006;47:10:1967-1975.

15. Birgelen CV, Hartmann M, MintZ GS. Relation between progression and regression of atherosclerotic left main coronary artery disease and serum cholesterol levels as assessed with serial long-term ( 12 months) follow-up intravascular ultrasound. Circulation. 2003;108:2757-2762.

16. Boden WE, O'Rourke RA, Teo KK. Courage Trial Research group. Optimal medical therapy with or without PCI for stable coronary disease. N Eng J Med. 2007;356:1503-1516. 